\title{
A new extended alpha power transformed family of distributions: properties, characterizations and an application to a data set in the insurance sciences
}

\author{
Zubair Ahmad $^{a}$, Eisa Mahmoudi ${ }^{1, a}$, G.G. Hamedani ${ }^{b}$ \\ ${ }^{a}$ Department of Statistics, Yazd University, Iran; \\ ${ }^{b}$ Department of Mathematical and Statistical Sciences, Marquette University, USA
}

\begin{abstract}
Heavy tailed distributions are useful for modeling actuarial and financial risk management problems. Actuaries often search for finding distributions that provide the best fit to heavy tailed data sets. In the present work, we introduce a new class of heavy tailed distributions of a special sub-model of the proposed family, called a new extended alpha power transformed Weibull distribution, useful for modeling heavy tailed data sets. Mathematical properties along with certain characterizations of the proposed distribution are presented. Maximum likelihood estimates of the model parameters are obtained. A simulation study is provided to evaluate the performance of the maximum likelihood estimators. Actuarial measures such as Value at Risk and Tail Value at Risk are also calculated. Further, a simulation study based on the actuarial measures is done. Finally, an application of the proposed model to a heavy tailed data set is presented. The proposed distribution is compared with some well-known (i) two-parameter models, (ii) three-parameter models and (iii) four-parameter models.
\end{abstract}

Keywords: alpha power transformation, Weibull distribution, family of distributions, actuarial measures, characterizations, maximum likelihood estimation, Monte Carlo simulation

\section{Introduction}

Statistical distributions play a vital role in modeling data in applied areas such as risk management, banking, economic, financial and actuarial sciences. However, the quality of the procedures primarily depends upon the assumed probability model of the phenomenon under consideration. Among applied fields, insurance data sets are usually positive (Klugman et al., 2012), right skewed (Lane, 2000), unimodal (Cooray and Ananda, 2005) and with heavy tails (Ibragimov and Prokhorov, 2017). Rightskewness may be adequately modeled by the skewed distributions (Bernardi et al., 2012). Therefore, a number of unimodal positively skewed parametric distributions have been deployed to model such data sets (Klugman et al., 2012; Eling, 2012; Bagnato and Punzo, 2013; García et al., 2014; Bakar et al., 2015; Kazemi and Noorizadeh, 2015; Adcock et al., 2015; Landsman et al., 2016; Reynkens et al., 2017; Punzo et al., 2017; Bhati and Ravi, 2018).

Among available literature, the Pareto, Weibull, and gamma are the promising distributions used widely for modeling insurance data sets. Unfortunately, due to the monotonically decreasing shape, the Pareto distribution often does not provide a best fit to many data sets. Weibull distribution is suitable for modeling small losses, but fails to provide best fit to large losses. In addition, gamma

\footnotetext{
${ }^{1}$ Corresponding author: Department of Statistics, Yazd University, P.O. Box 89175-741, Yazd, Iran.

E-mail: emahmoudi@yazd.ac.ir
}

Published 31 January 2021 / journal homepage: http://csam.or.kr

(c) 2021 The Korean Statistical Society, and Korean International Statistical Society. All rights reserved. 
distribution is also popular because it does not have a closed form expression of cumulative distribution function (cdf). Consequently, introducing new distributions to address these problems and cater heavy tailed data is an interesting research topic that is quite rich and growing rapidly. Therefore, researchers are often in search of finding more flexible distributions. This has been done through many different approaches such as (i) transformation method, (ii) composition of two or more distributions, (iii) compounding of distributions, and (iv) finite mixture of distributions, for details see Ahmad et al. (2019a).

Among the prominent methods, Mahdavi and Kundu (2017) recently proposed a new method for introducing statistical distributions via the cdf given by

$$
G\left(x ; \alpha_{1}, \xi\right)=\frac{\alpha_{1}^{F(x ; \xi)}-1}{\alpha_{1}-1}, \quad \alpha_{1}>0, \alpha_{1} \neq 1, x \in \mathbb{R},
$$

where $F(x ; \xi)$ is the distribution function of the baseline random variable depending on the vector parameter $\xi \in \mathbb{R}$, and $\alpha$ is the additional parameter.

Ahmad et al. (2019b) proposed another method, called extended alpha power transformed (ExAPT) family of distributions with cdf given by

$$
G\left(x ; \alpha_{1}, \xi\right)=\frac{\alpha_{1}^{F(x ; \xi)}-e^{F(x ; \xi)}}{\alpha_{1}-e}, \quad \alpha_{1}>0, \alpha_{1} \neq e, x \in \mathbb{R} .
$$

The Ex-APT family is an extension of (1.1) by introducing the exponent term, which is a constant quantity. Because of the constant term, the Ex-APT family approach is not a flexible extension of (1.1). In this article, we introduce another new extension of (1.1), called new Ex-APT (NEx-APT) family by introducing an additional parameter $\beta \in \mathbb{R}^{+}$. A random variable $X$ follows the NEx-APT distribution, if its cdf is given by

$$
G\left(x ; \alpha_{1}, \beta, \xi\right)=\frac{\alpha_{1}^{F(x ; \xi)}-[1-\bar{\beta} F(x, \xi)]}{\alpha_{1}-\beta}, \quad \alpha_{1}, \beta>0, \alpha_{1}>\beta, x \in \mathbb{R},
$$

where, $\bar{\beta}=1-\beta$. For $\beta=1$, the NEx-APT method captures the characteristics of (1.1) and for $\alpha_{1}=1$, the NEx-APT approach offers the baseline distribution characteristics. The probability density function (pdf) corresponding to (1.2) is given by

$$
g\left(x ; \alpha_{1}, \beta, \xi\right)=\frac{f(x, \xi)\left[\log \left(\alpha_{1}\right) \alpha_{1}^{F(x, \xi)}+\bar{\beta}\right]}{\alpha_{1}-\beta}, \quad x \in \mathbb{R} .
$$

The new pdf is most tractable when $F(x, \xi)$ and $f(x, \xi)$ have simple analytical expressions. A random variable $X$ with pdf (1.3) is represented by $X \sim \operatorname{NEx}$-APT $\left(x ; \alpha_{1}, \beta, \xi\right)$. Furthermore, the dependence on the vector of the parameters is omitted and $G(x)=G\left(x ; \alpha_{1}, \beta, \xi\right)$ will be used for efficiency. The proposed method is very interesting with a closed form for the cdf and capable of modeling heavy tailed insurance data sets.

The rest of this work is as follows. In Section 2, we introduce a special sub-case of (1.3), called a NEx-APT Weibull (NEx-APTW) distribution by considering Weibull model as a parent distribution. Some mathematical properties are derived in Section 3. Certain characterizations of the proposed distribution are provided in Section 4. Maximum likelihood estimation and Monti Carlo simulation study are provided in Section 5. Section 6 offers the computation of the actuarial measures and simulation study based on these measures. A heavy tailed real-life application from the insurance sciences is discussed in Section 7. Here the proposed model is compared with some prominent distributions. Finally, the article is concluded in Section 8. 


\section{Sub-model and its special cases}

In this section, we introduce a sub-model of the (1.2) and discuss its special cases.

\subsection{A new extended alpha power transformed Weibull distribution}

Let $F(x ; \xi)$ and $f(x ; \xi)$ be the cdf and pdf of the two parameters Weibull distribution given by $F(x ; \xi)=$ $1-e^{-\gamma x^{\alpha}}, x \geq 0, \alpha, \gamma>0$, and $f(x ; \xi)=\alpha \gamma x^{\alpha-1} e^{-\gamma x^{\alpha}}$, respectively, where $\xi=(\alpha, \gamma)$. Then, the cdf of the NEx-APTW distribution has the following expression

$$
G(x)=\frac{\alpha_{1}^{\left(1-e^{-\gamma x^{\alpha}}\right)}-\left[1-\bar{\beta}\left(1-e^{-\gamma x^{\alpha}}\right)\right]}{\alpha_{1}-\beta}, \quad \alpha_{1}, \beta, \alpha, \gamma>0, \alpha_{1}>\beta, x \geq 0 .
$$

The pdf corresponding (2.1) is given by

$$
g(x)=\frac{\alpha \gamma x^{\alpha-1} e^{-\gamma x^{\alpha}}\left[\log \left(\alpha_{1}\right) \alpha_{1}^{\left(1-e^{-\gamma x^{\alpha}}\right)}+\bar{\beta}\right]}{\alpha_{1}-\beta}, \quad x>0 .
$$

The survival function (sf) and hazard function (hf) of NEx-APTW distribution are respectively, given by

$$
\begin{aligned}
& S(x)=\frac{\alpha_{1}-\beta-\left\{\alpha_{1}^{\left(1-e^{-\gamma x^{\alpha}}\right)}-\left[1-\bar{\beta}\left(1-e^{-\gamma x^{\alpha}}\right)\right]\right\}}{\alpha_{1}-\beta}, \quad x \geq 0, \\
& h(x)=\frac{\alpha \gamma x^{\alpha-1} e^{-\gamma x^{\alpha}}\left[\log \left(\alpha_{1}\right) \alpha_{1}^{\left(1-e^{-\gamma x^{\alpha}}\right)}+\bar{\beta}\right]}{\alpha_{1}-\beta-\left\{\alpha_{1}^{\left(1-e^{-\gamma x^{\alpha}}\right)}-\left[1-\bar{\beta}\left(1-e^{-\gamma x^{\alpha}}\right)\right]\right\}}, \quad x>0 .
\end{aligned}
$$

Figure 1 sketches different plots for the density function of the NEx-APTW distribution.

\subsection{Special cases of NEx-APTW distribution}

Let $X$ have the NEx-APTW distribution with parameters $\left(\alpha_{1}, \beta, \alpha, \gamma\right)$. Then, the distribution of $X$ reduces to:

1. Alpha power transformed Weibull (APTW) distribution, if $\beta=1$.

2. Alpha power transformed exponential (APTE) distribution, if $\alpha=1$ and $\beta=1$.

3. Weibull distribution, if $\alpha_{1}=1$.

4. Rayleigh distribution, if $\alpha_{1}=1$ and $\alpha=2$.

5. Exponential distribution if $\alpha_{1}=1$ and $\alpha=1$.

6. One parameter Weibull distribution, if $\alpha_{1}=1$ and $\gamma=1$.

7. One parameter New extended alpha power transformed Weibull, if $\gamma=1$. (New)

8. New extended alpha power transformed Rayleigh, if $\alpha=2$. (New)

9. New extended alpha power transformed exponential, if $\alpha=1$. (New) 

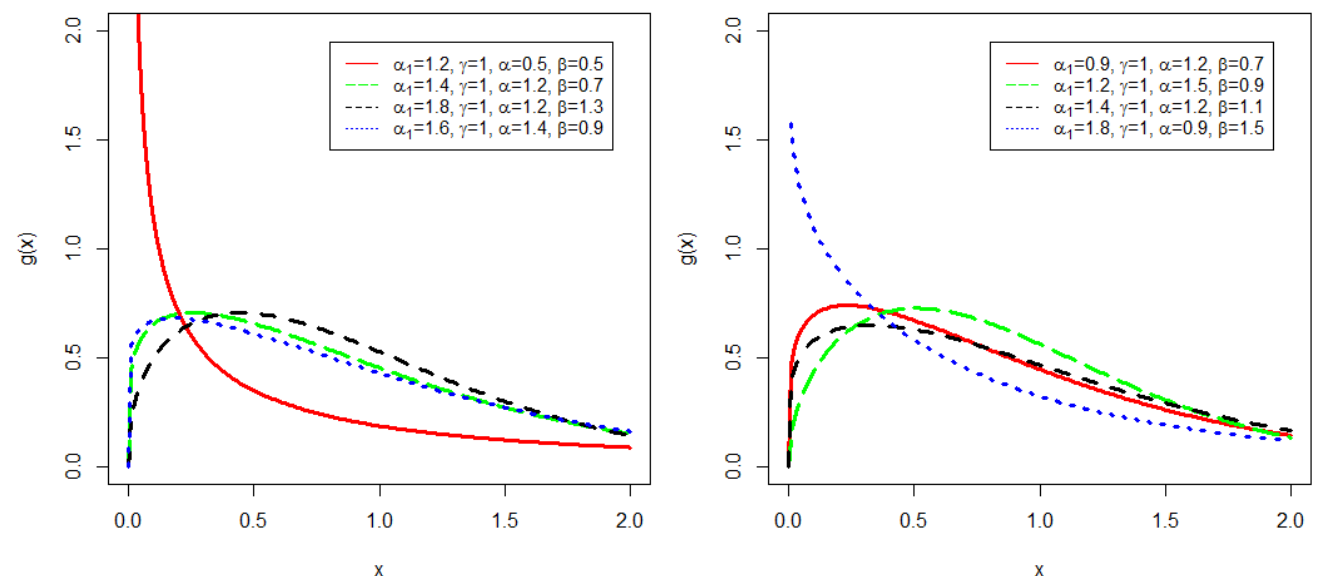

Figure 1: Different plots for the density function of the NEx-APTW distribution. NEx-APTW = new extended alpha power transformed Weibull

\section{Mathematical properties}

In this section, we derive some mathematical properties of the NEx-APT distribution, such as quantile function, moments and moment generating function.

\subsection{Quantile function}

The quantile function of the NEx-APT distribution, denoted by $Q(u)$, can be obtained by inverting its cdf (1.2). Therefore, we have

$$
x_{q}=Q(u)=G^{-1}(u)=F^{-1}(t),
$$

where $t$ is the solution of the expression $\alpha_{1}^{t}+\bar{\beta} t-u\left(\alpha_{1}-\beta\right)-1=0$, and $u \in(0,1)$. The nonlinear expression (3.1) can be used to obtain random numbers for the NEx-APT distribution.

\subsection{Moments}

In this sub-section, we intend to derive the moments and the moment generating function of the NExAPT distribution. Let $X$ follow (1.3), then, the $r^{\text {th }}$ moment of $X$ is derived as

$$
\mu_{r}^{\prime}=\int_{-\infty}^{\infty} x^{r} g\left(x ; \alpha_{1}, \beta, \xi\right) d x
$$

and using (1.3) in (3.2), we have

$$
\mu_{r}^{\prime}=\int_{-\infty}^{\infty} x^{r} \frac{f(x, \xi)\left[\log \left(\alpha_{1}\right) \alpha_{1}^{F(x, \xi)}+\bar{\beta}\right]}{\alpha_{1}-\beta} d x .
$$

The function $\alpha_{1}^{x}$ has the Maclaurin series given by

$$
\alpha_{1}^{x}=\sum_{n=0}^{\infty}\left(\log \left(\alpha_{1}\right)\right)^{n} \frac{x^{n}}{n !} .
$$


Taking $x=F(x ; \xi)$ in (3.4), we get

$$
\alpha_{1}^{x}=\sum_{n=0}^{\infty}\left(\log \left(\alpha_{1}\right)\right)^{n} \frac{F(x ; \xi)^{n}}{n !}
$$

Using (3.5) in (3.3), we have

$$
\mu_{r}^{\prime}=\sum_{n=0}^{\infty}\left(\log \left(\alpha_{1}\right)\right)^{n+1} \frac{\Lambda_{r, n}}{\left(\alpha_{1}-\beta\right) n !}+\frac{\bar{\beta} \Lambda_{r}}{\left(\alpha_{1}-\beta\right)},
$$

where $\Lambda_{r, n}=\int_{-\infty}^{\infty} x^{r} f(x, \xi) F(x ; \xi)^{n} d x$ and $\Lambda_{r}=\int_{-\infty}^{\infty} x^{r} f(x, \xi) d x$.

Furthermore, the moment generating function of the NEx-APT random variable $X, M_{X}(t)$, is given by

$$
M_{X}(t)=\sum_{r, n=0}^{\infty}\left(\log \left(\alpha_{1}\right)\right)^{n+1} \frac{t^{r} \Lambda_{r, n}}{\left(\alpha_{1}-\beta\right) n ! r !}+\sum_{r=0}^{\infty} \frac{t^{r} \bar{\beta} \Lambda_{r}}{\left(\alpha_{1}-\beta\right) n ! r !}
$$

\section{Characterizations of NEx-APT distribution}

In this section, we present certain characterizations of the NEx-APT distribution in the following directions: (i) based on a simple relationship between two truncated moments and (ii) in terms of the reverse hazard function. It should be mentioned that for the characterization (i) the cdf is not required to have a closed form.

We present our characterizations (i)-(ii) in two subsections.

\subsection{Characterizations based on two truncated moments}

In this subsection, we present characterizations of NEx-APT distribution in terms of a simple relationship between two truncated moments. The first characterization result employs a theorem due to Glänzel (1987), see Theorem 4.1 below. Note that the result holds also when the interval $H$ is not closed. Moreover, in it could be also applied when the cdf $F$ does not have a closed form. As shown in Glänzel (1990), this characterization is stable in the sense of weak convergence.

Theorem 1. Let $(\Omega, \mathcal{F}, \mathbf{P})$ be a given probability space and let $H=[d, e]$ be an interval for some $d<e(d=-\infty, e=\infty$ might as well be allowed $)$. Let $X: \Omega \rightarrow H$ be a continuous random variable with the distribution function $G$ and let $q_{1}$ and $q_{2}$ be two real functions defined on $H$ such that

$$
\mathbf{E}\left[q_{2}(X) \mid X \geq x\right]=\mathbf{E}\left[q_{1}(X) \mid X \geq x\right] \eta(x), \quad x \in H,
$$

is defined with some real function $\eta$. Assume that $q_{1}, q_{2} \in C^{1}(H), \eta \in C^{2}(H)$ and $G$ is twice continuously differentiable and strictly monotone function on the set $H$. Finally, assume that the equation $\eta q_{1}=q_{2}$ has no real solution in the interior of $H$. Then $G$ is uniquely determined by the functions $q_{1}, q_{2}$, and $\eta$, particularly

$$
G(x)=\int_{a}^{x} C\left|\frac{\eta^{\prime}(u)}{\eta(u) q_{1}(u)-q_{2}(u)}\right| \exp (-s(u)) d u,
$$

where the function $s$ is a solution of the differential equation $s^{\prime}=\eta^{\prime} q_{1} /\left(\eta q_{1}-q_{2}\right)$ and $C$ is the normalization constant, such that $\int_{H} d G=1$. 
Proposition 1. Let $X: \Omega \rightarrow \mathbb{R}$ be a continuous random variable and let $q_{1}(x)=\left[\log \left(\alpha_{1}\right) \alpha_{1}^{F(x ; \xi)}+\right.$ $\bar{\beta}]^{-1}$ and $q_{2}(x)=q_{1}(x) F(x ; \xi)$ for $x \in \mathbb{R}$. The random variable $X$ has $p d f(1.3)$ only if the function $\eta$ defined in Theorem 1 has the form

$$
\eta(x)=\frac{1}{2}[1+F(x ; \xi)], \quad x \in \mathbb{R} .
$$

Proof: Let $X$ be a random variable with pdf (1.3), then

$$
\begin{aligned}
(1-G(x)) E\left[q_{1}(X) \mid X \geq x\right] & =\int_{x}^{\infty} q_{1}(x) g\left(x ; \alpha_{1}, \beta, \xi\right) d x \\
& =\frac{1}{\alpha_{1}-\beta} \int_{x}^{\infty} f(x ; \xi) d x \\
& =\frac{1}{\alpha_{1}-\beta}(1-F(x)), \quad x \in \mathbb{R}
\end{aligned}
$$

and

$$
\begin{aligned}
(1-G(x)) E\left[q_{2}(X) \mid X \geq x\right] & =\int_{x}^{\infty} q_{2}(x) g\left(x ; \alpha_{1}, \beta, \xi\right) d x \\
& =\frac{1}{\alpha_{1}-\beta} \int_{x}^{\infty} f(x ; \xi) F(x ; \xi) d x \\
& =\frac{1}{2\left(\alpha_{1}-\beta\right)}\left(1-F(x)^{2}\right), \quad x \in \mathbb{R},
\end{aligned}
$$

and finally

$$
\begin{aligned}
\eta(x) q_{1}(x)-q_{2}(x) & =\frac{1}{2}(1+F(x)) q_{1}(x)-q_{1}(x) F(x) \\
& =\frac{q_{1}(x)}{2}\{1+F(x)-2 F(x)\} \\
& =\frac{q_{1}(x)}{2}\{1-F(x)\}, \quad \text { for } x \in \mathbb{R} .
\end{aligned}
$$

Conversely, if $\eta$ is given as above, then

$$
s^{\prime}(x)=\frac{\eta^{\prime}(x) q_{1}(x)}{\eta(x) q_{1}(x)-q_{2}(x)}=\frac{f(x ; \xi)}{1-F(x ; \xi)}, \quad x \in \mathbb{R},
$$

therefore,

$$
s(x)=-\log [1-F(x ; \xi)], \quad x \in \mathbb{R} .
$$

Now, in view of Theorem $1, X$ has density (1.3).

Corollary 1. Let $X: \Omega \rightarrow \mathbb{R}$ be a continuous random variable and let $q_{1}(x)$ be as in Proposition 1. The pdf of $X$ is (1.3) only if there exist functions $q_{2}$ and $\eta$ defined in Theorem 1 satisfying the differential equation

$$
\frac{\eta^{\prime}(x) q_{1}(x)}{\eta(x) q_{1}(x)-q_{2}(x)}=\frac{f(x ; \xi)}{1-F(x ; \xi)}, \quad x \in \mathbb{R} .
$$


Corollary 2. The general solution of the differential equation in Corollary 1 is

$$
\eta(x)=[1-F(x ; \xi)]^{-1}\left[-\int f(x ; \xi)\left(q_{1}(x)\right)^{-1} q_{2}(x)+D\right],
$$

where $D$ is a constant. Note that a set of functions satisfying the above differential equation is given in Proposition 1 with $D=1 / 2$. However, it should be also noted that there are other triplets $\left(q_{1}, q_{2}, \eta\right)$ satisfying the conditions of Theorem 1.

\subsection{Characterization in terms of the reverse hazard function}

The reverse hazard function, $r_{G}$, of a twice differentiable distribution function, $G$, is defined as

$$
r_{G}(x)=\frac{g(x)}{G(x)}, \quad x \in \text { support of } G .
$$

In this subsection, we present a characterization of the NEx-APT distribution in terms of the reverse hazard function.

Proposition 2. Let $X: \Omega \rightarrow \mathbb{R}$ be a continuous random variable. The random variable $X$ has pdf (1.3) only if its reverse hazard function $r_{G}(x)$ satisfies the following differential equation.

$$
r_{G}^{\prime}(x)-\frac{f^{\prime}(x ; \xi)}{f(x ; \xi)} r_{G}(x)=f(x ; \xi) \frac{d}{d x}\left\{\frac{\left[\log (\alpha) \alpha^{F(x ; \xi)}+\bar{\beta}\right]}{\alpha^{F(x ; \xi)}-[1-\bar{\beta} F(x ; \xi)]}\right\}, \quad x \in \mathbb{R} .
$$

Proof: Is straightforward and hence omitted.

\section{Estimation and simulation}

In this section, we estimate the parameters of the NEx-APT distribution via the method of maximum likelihood and provide Monti Carlo simulation to evaluate the performance of these estimators.

\subsection{Maximum likelihood estimation}

Let $x_{1}, x_{2}, \ldots, x_{n}$ be observed values of a random sample from NEx-APT distribution with parameters $\left(\alpha_{1}, \beta, \xi\right)$. The log-likelihood function of this sample is

$$
\ell(\Theta)=n \log \left(\alpha_{1}-\beta\right)+\sum_{i=1}^{n} \log f\left(x_{i} ; \xi\right)+\sum_{i=1}^{n} \log \left\{\log \left(\alpha_{1}\right) \alpha_{1}^{F\left(x_{i} ; \xi\right)}+\bar{\beta}\right\},
$$

where, $\Theta=\left(\alpha_{1}, \beta, \xi\right)^{T}$ be the $3 \times 1$ parameter vector. The partial derivatives of (5.1) are

$$
\begin{aligned}
& \frac{\partial \ell(\Theta)}{\partial \alpha_{1}}=\frac{n}{\left(\alpha_{1}-\beta\right)}+\sum_{i=1}^{n} \frac{\left\{\log \left(\alpha_{1}\right) F\left(x_{i} ; \xi\right)+1\right\} \alpha_{1}^{F\left(x_{i} ; \xi\right)-1}}{\left\{\log \left(\alpha_{1}\right) \alpha_{1}^{F\left(x_{i} ; \xi\right)}+\bar{\beta}\right\}}, \\
& \frac{\partial \ell(\Theta)}{\partial \beta}=\frac{-n}{\left(\alpha_{1}-\beta\right)}-\sum_{i=1}^{n} \frac{1}{\left\{\log \left(\alpha_{1}\right) \alpha_{1}^{F\left(x_{i} ; \xi\right)}+\bar{\beta}\right\}},
\end{aligned}
$$


and

$$
\frac{\partial \ell(\Theta)}{\partial \xi}=\sum_{i=1}^{n} \frac{\partial f\left(x_{i} ; \xi\right) / \partial \xi}{f\left(x_{i} ; \xi\right)}+\sum_{i=1}^{n} \frac{\left(\log \left(\alpha_{1}\right)\right)^{2} \alpha_{1}^{F\left(x_{i} ; \xi\right)} \partial F\left(x_{i} ; \xi\right) / \partial \xi}{\left\{\log \left(\alpha_{1}\right) \alpha_{1}^{F\left(x_{i} ; \xi\right)}+\bar{\beta}\right\}} .
$$

Setting $\left(\partial / \partial \alpha_{1}\right) \ell(\Theta),(\partial / \partial \beta) \ell(\Theta)$, and $(\partial / \partial \xi) \ell(\Theta)$ equal to zero and solving numerically these expressions simultaneously, yields the maximum likelihood estimates of $\left(\alpha_{1}, \beta, \xi\right)$.

\subsection{Asymptotic confidence bounds}

In this subsection, we derive the asymptotic confidence intervals of the unknown parameters of the NEx-APT distributions. The simplest large sample approach is to assume that the maximum likelihood estimators $\left(\hat{\alpha_{1}}, \hat{\beta}, \hat{\xi}\right)$ are approximately multivariate normal with mean $\left(\alpha_{1}, \beta, \xi\right)$ and covariance matrix $I_{0}^{-1}$, where $I_{0}^{-1}$ is the inverse of the observed information matrix defined by

$$
I_{0}^{-1}=-E\left[\begin{array}{lll}
\frac{\partial^{2} \ell(\Theta)}{\partial \alpha_{1}^{2}} & \frac{\partial^{2} \ell(\Theta)}{\partial \alpha_{1} \partial \beta} & \frac{\partial^{2} \ell(\Theta)}{\partial \alpha_{1} \partial \xi} \\
\frac{\partial^{2} \ell(\Theta)}{\partial \beta \partial \alpha_{1}} & \frac{\partial^{2} \ell(\Theta)}{\partial \beta^{2}} & \frac{\partial^{2} \ell(\Theta)}{\partial \beta \partial \xi} \\
\frac{\partial^{2} \ell(\Theta)}{\partial \xi \partial \alpha_{1}} & \frac{\partial^{2} \ell(\Theta)}{\partial \xi \partial \beta} & \frac{\partial^{2} \ell(\Theta)}{\partial \xi^{2}}
\end{array}\right]^{-1}=\left[\begin{array}{lll}
\operatorname{var}\left(\hat{\alpha}_{1}\right) & \operatorname{cov}\left(\hat{\alpha}_{1}, \hat{\beta}\right) & \operatorname{cov}\left(\hat{\alpha}_{1}, \hat{\xi}\right) \\
\operatorname{cov}\left(\hat{\beta}, \hat{\alpha}_{1}\right) & \operatorname{var}(\hat{\beta}) & \operatorname{cov}(\beta, \hat{\xi}) \\
\operatorname{cov}\left(\hat{\xi}, \hat{\alpha}_{1}\right) & \operatorname{cov}(\hat{\xi}, \beta) & \operatorname{var}(\hat{\xi})
\end{array}\right] .
$$

The second partial derivatives included in $I_{0}^{-1}$ are given as follows

$$
\begin{aligned}
\frac{\partial^{2} \ell(\Theta)}{\partial \xi \partial \alpha_{1}}= & \sum_{i=1}^{n} \frac{\alpha_{1}^{F(x ; \xi)}\left(\log \alpha_{1}\right)\left\{\alpha_{1}^{F(x ; \xi)-1}+\bar{\beta}\left(\log \alpha_{1}\right)(\partial F(x ; \xi) / \partial \xi)+2 \bar{\beta} \alpha_{1}^{-1}\right\}}{\left(\left(\log \alpha_{1}\right) \alpha_{1}^{F(x ; \xi)}+\bar{\beta}\right)^{2}}, \\
\frac{\partial^{2} \ell(\Theta)}{\partial \alpha_{1}^{2}}= & \sum_{i=1}^{n} \frac{\alpha_{1}^{F(x ; \xi)-1}\left\{\left(\log \alpha_{1}\right) \bar{\beta} F(x ; \xi)^{2}+\bar{\beta}+\beta \alpha_{1}^{-1} \alpha_{1}^{F(x ; \xi)-1}(F(x ; \xi)-1)-\alpha_{1}^{F(x ; \xi)-1}\right\}}{\left(\left(\log \alpha_{1}\right) \alpha_{1}^{F(x ; \xi)}+\bar{\beta}\right)^{2}}, \\
\frac{\partial^{2} \ell(\Theta)}{\partial \xi^{2}}= & \sum_{i=1}^{n} \frac{\alpha_{1}^{F(x ; \xi)}(\partial F(x ; \xi) / \partial \xi)\left\{\left(\log \alpha_{1}\right) \alpha_{1}^{F(x ; \xi)}+\bar{\beta}\left(\log \alpha_{1}\right)^{3}(\partial F(x ; \xi) / \partial \xi)+\bar{\beta}\right\}}{\left(\left(\log \alpha_{1}\right) \alpha_{1}^{F(x ; \xi)}+\bar{\beta}\right)^{2}} \\
& +\sum_{i=1}^{n} \frac{f(x ; \xi)\left(\partial^{2} f(x ; \xi) / \partial \xi^{2}\right)-(\partial f(x ; \xi) / \partial \xi)^{2}}{(f(x ; \xi))^{2}} \\
\frac{\partial^{2} \ell(\Theta)}{\partial \alpha_{1} \partial \beta}= & \frac{n}{\left(\alpha_{1}-\beta\right)}+\sum_{i=1}^{n} \frac{\left\{\left(\log \alpha_{1}\right) F(x ; \xi)+1\right\} \alpha_{1}^{F(x ; \xi)-1}}{\left(\left(\log \alpha_{1}\right) \alpha_{1}^{F(x ; \xi)}+\bar{\beta}\right)^{2}}, \\
\frac{\partial^{2} \ell(\Theta)}{\partial \beta^{2}}= & -\frac{n}{\left(\alpha_{1}-\beta\right)^{2}}-\sum_{i=1}^{n} \frac{1}{\left(\left(\log \alpha_{1}\right) \alpha_{1}^{F(x ; \xi)}+\bar{\beta}\right)^{2}},
\end{aligned}
$$

and

$$
\frac{\partial^{2} \ell(\Theta)}{\partial \beta \partial \xi}=\sum_{i=1}^{n} \frac{\left(\log \alpha_{1}\right) \alpha_{1}^{F(x ; \xi)}(\partial F(x ; \xi) / \partial \xi)}{\left(\left(\log \alpha_{1}\right) \alpha_{1}^{F(x ; \xi)}+\bar{\beta}\right)^{2}} .
$$



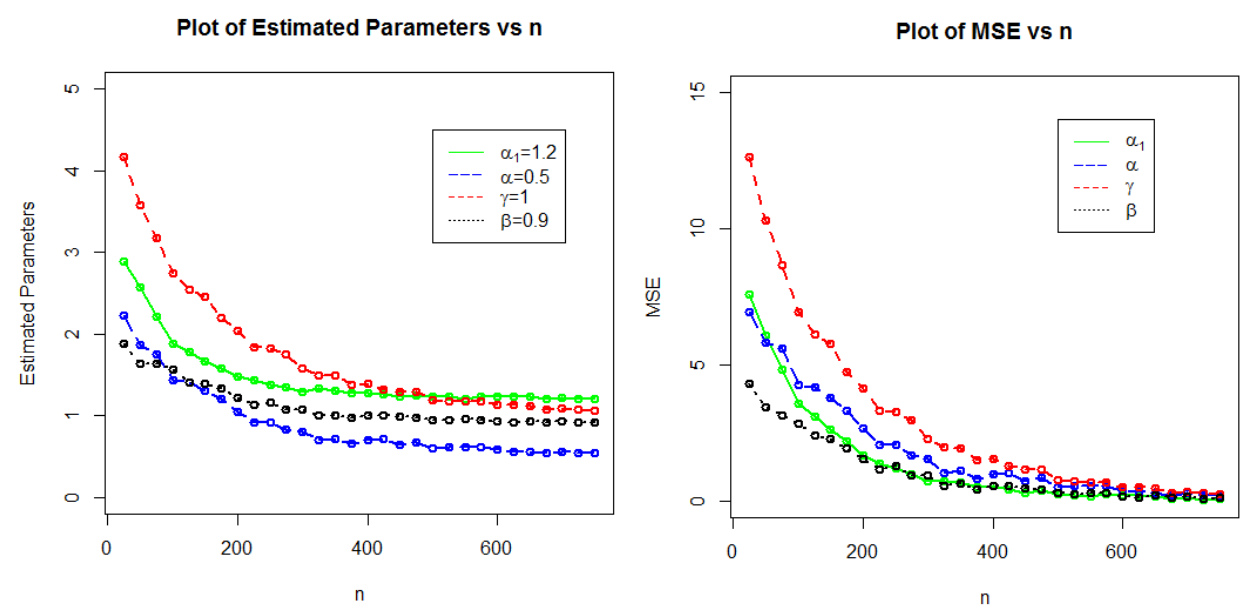

Figure 2: Plots of the estimated parameters and MSEs of the new extended alpha power transformed Weibull distribution for Table 1. MSE = mean squared error.

The above approach is used to derive the $(1-\delta) 100 \%$ confidence intervals for the parameters $\alpha_{1}, \beta$, and $\xi$ as in the following forms $\hat{\alpha}_{1} \pm Z_{\delta / 2} \sqrt{\operatorname{var}\left(\hat{\alpha}_{1}\right)}, \hat{\beta} \pm Z_{\delta / 2} \sqrt{\operatorname{var}(\hat{\beta})}$, and $\hat{\xi} \pm Z_{\delta / 2} \sqrt{\operatorname{var}(\hat{\xi})}$, where $Z_{\delta / 2}$ is the upper $(\delta / 2)^{\text {th }}$ percentile of the standard normal distribution.

\subsection{Simulation study of the NEx-APTW distribution}

In order to evaluate the performances of the maximum likelihood estimators of the $\left(\alpha_{1}, \beta, \xi\right)$, a simulation study is conducted. The process is carried out as:

1. We generate $N=750$ samples of sizes $n=25,50, \ldots, 750$ from the NEx-APTW distribution.

2. Initial values for the parameters are selected as given in Table 1 .

3. Compute the biases and MSEs given by $\operatorname{Bias}(\hat{w})=(1 / 750) \sum_{i=1}^{750}\left(\hat{w}_{i}-w\right)$ and $\operatorname{MSE}(\hat{w})=(1 / 750)$ $\sum_{i=1}^{750}\left(\hat{w}_{i}-w\right)^{2}$, for $w=\left(\alpha_{1}, \beta, \xi\right)$, respectively.

\section{Actuarial measures of the NEx-APTW distribution}

One of the most important tasks of actuaries is to evaluate the exposure to market risk in a portfolio of instruments, which arise from changes in underlying variables such as prices of equity, interest rates or exchange rates. In this section, we calculate two well-known and important risk measures Value at Risk (VaR) and Tail VaR (TVaR) for the proposed distribution, which play a crucial role in portfolio optimization under uncertainty. Furthermore, based on these measures, a simulation study is performed and we show that the proposed distribution has heavier tails the Weibull and exponentiated Weibull (EW) distributions.

\subsection{Value at risk}

In the context of actuarial sciences, the measure VaR is widely used by practitioners as a standard financial market risk. It is also known as the quantile risk measure or quantile premium principle. VaR 
Table 1: Simulation results for different combination of parameters values of new extended alpha power transformed Weibull distribution

\begin{tabular}{|c|c|c|c|c|}
\hline \multicolumn{5}{|c|}{$\alpha_{1}=1.2, \alpha=0.5, \gamma=1, \beta=0.9$} \\
\hline$n$ & Parameters & MLE & Bias & MSE \\
\hline \multirow{4}{*}{25} & $\hat{\alpha_{1}}$ & 2.905 & 1.705 & 7.485 \\
\hline & $\hat{\alpha}$ & 2.197 & 1.697 & 6.860 \\
\hline & $\hat{\gamma}$ & 4.098 & 3.098 & 12.38 \\
\hline & $\hat{\beta}$ & 1.718 & 0.818 & 3.805 \\
\hline \multirow{4}{*}{50} & $\hat{\alpha_{1}}$ & 2.550 & 1.350 & 6.123 \\
\hline & $\hat{\alpha}$ & 1.914 & 1.414 & 5.932 \\
\hline & $\hat{\gamma}$ & 3.569 & 2.569 & 10.25 \\
\hline & $\hat{\beta}$ & 1.682 & 0.782 & 3.525 \\
\hline \multirow{4}{*}{100} & $\hat{\alpha_{1}}$ & 1.945 & 0.745 & 3.829 \\
\hline & $\hat{\alpha}$ & 1.565 & 1.065 & 4.843 \\
\hline & $\hat{\gamma}$ & 2.825 & 1.825 & 7.272 \\
\hline & $\hat{\beta}$ & 1.603 & 0.703 & 3.020 \\
\hline \multirow{4}{*}{150} & $\hat{\alpha_{1}}$ & 1.792 & 0.592 & 2.957 \\
\hline & $\hat{\alpha}$ & 1.225 & 0.725 & 3.381 \\
\hline & $\hat{\gamma}$ & 2.400 & 1.400 & 5.543 \\
\hline & $\hat{\beta}$ & 1.382 & 0.482 & 2.127 \\
\hline \multirow{4}{*}{250} & $\hat{\alpha_{1}}$ & 1.398 & 0.198 & 1.127 \\
\hline & $\hat{\alpha}$ & 0.844 & 0.344 & 1.718 \\
\hline & $\hat{\gamma}$ & 1.695 & 0.695 & 2.767 \\
\hline & $\hat{\beta}$ & 1.084 & 0.184 & 0.951 \\
\hline \multirow{4}{*}{350} & $\hat{\alpha_{1}}$ & 1.270 & 0.070 & 0.545 \\
\hline & $\hat{\alpha}$ & 0.739 & 0.239 & 1.192 \\
\hline & $\hat{\gamma}$ & 1.452 & 0.452 & 1.790 \\
\hline & $\hat{\beta}$ & 1.035 & 0.865 & 0.681 \\
\hline \multirow{4}{*}{450} & $\hat{\alpha_{1}}$ & 1.245 & 0.045 & 0.309 \\
\hline & $\hat{\alpha}$ & 0.590 & 0.090 & 0.495 \\
\hline & $\hat{\gamma}$ & 1.266 & 0.266 & 1.044 \\
\hline & $\hat{\beta}$ & 0.948 & 0.048 & 0.268 \\
\hline \multirow{4}{*}{550} & $\hat{\alpha_{1}}$ & 1.216 & 0.016 & 0.157 \\
\hline & $\hat{\alpha}$ & 0.578 & 0.078 & 0.410 \\
\hline & $\hat{\gamma}$ & 1.169 & 0.169 & 0.671 \\
\hline & $\hat{\beta}$ & 0.929 & 0.029 & 0.196 \\
\hline \multirow{4}{*}{650} & $\hat{\alpha_{1}}$ & 1.226 & 0.026 & 0.143 \\
\hline & $\hat{\alpha}$ & 0.549 & 0.049 & 0.244 \\
\hline & $\hat{\gamma}$ & 1.183 & 0.083 & 0.331 \\
\hline & $\hat{\beta}$ & 0.934 & 0.014 & 0.101 \\
\hline \multirow{4}{*}{750} & $\hat{\alpha_{1}}$ & 1.194 & 0.005 & 0.031 \\
\hline & $\hat{\alpha}$ & 0.520 & 0.040 & 0.189 \\
\hline & $\hat{\gamma}$ & 1.052 & 0.052 & 0.209 \\
\hline & $\hat{\beta}$ & 0.920 & 0.010 & 0.096 \\
\hline
\end{tabular}

MLE = maximum likelihood estimators; MSE = mean squared error.

of a random variable $X$ is the $q^{\text {th }}$ quantile of its cdf and specified with a given degree of confidence say $q$ (typically 90, 95, or 99\%), see Artzner (1999). If $X$ has pdf (1.3), then the VaR of $X$ is given by

$$
x_{q}=F^{-1}(t),
$$

where $t$ is the solution of the expression $\alpha_{1}^{t}+\bar{\beta} t-q\left(\alpha_{1}-\beta\right)-1=0$. 


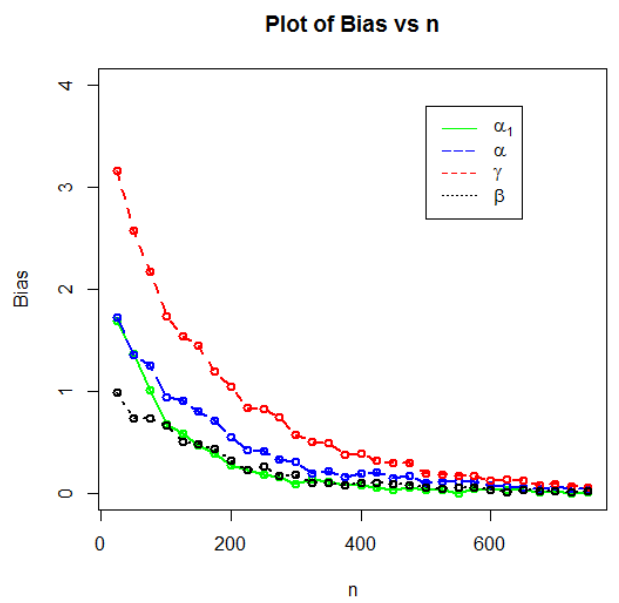

Figure 3: Plot of the biases of the parameters of the new extended alpha power transformed Weibull distribution for Table 1.

\subsection{Tail value at risk}

Another important measure is TVaR, also known as conditional tail expectation (CTE) used to quantify the expected value of the loss given that an event outside a given probability level has occurred. Let $X$ follow the NEx-APTW distribution, then the TVaR of $X$ is defined as

$$
\mathrm{TVaR}=\frac{1}{1-q} \int_{\mathrm{VaR}_{q}}^{\infty} x g\left(x ; \alpha_{1}, \beta, \xi\right) d x .
$$

Using (1.3) in (6.2), we have

$$
\operatorname{TVaR}=\frac{\alpha \gamma}{(1-q)\left(\alpha_{1}-\beta\right)} \int_{\operatorname{VaR}_{q}}^{\infty} x^{\alpha+1-1} e^{-\gamma x^{\alpha}}\left[\log \left(\alpha_{1}\right) \alpha_{1}^{\left(1-e^{-\gamma x^{\alpha}}\right)}+\bar{\beta}\right] d x .
$$

Using the series $\alpha_{1}^{x}=\sum_{n=0}^{\infty}\left(\log \left(\alpha_{1}\right)\right)^{n}\left(x^{n} / n !\right)$, we have

$$
\operatorname{TVaR}=\frac{\alpha \gamma}{(1-q)\left(\alpha_{1}-\beta\right)} \int_{\operatorname{VaR}_{q}}^{\infty} x^{\alpha+1-1} e^{-\gamma x^{\alpha}}\left[\sum_{i=1}^{\infty} \frac{\left(\log \left(\alpha_{1}\right)\right)^{i+1}}{n !}\left(1-e^{-\gamma x^{\alpha}}\right)^{n}+\bar{\beta}\right] d x
$$

or

$$
\operatorname{TVaR}=\frac{\sum_{i, n=0}^{\infty} \frac{\left(\log \left(\alpha_{1}\right)\right)^{i+1}\left(\begin{array}{c}
n \\
j
\end{array}\right)}{n !} \Gamma\left(\frac{1}{\alpha}+1, \gamma(j+1)\left(\operatorname{VaR}_{q}\right)^{\alpha}\right)}{(1-q)\left(\alpha_{1}-\beta\right)}+\bar{\beta} \frac{\Gamma\left(\frac{1}{\alpha}+1, \gamma\left(\operatorname{VaR}_{q}\right)^{\alpha}\right)}{(1-q)\left(\alpha_{1}-\beta\right)} .
$$

Based on actuarial measures, a simulation study is performed for the Weibull, exponentiated (EW) and proposed model for the selected parameters values. A model with higher values of the Risk measures (VaR and TVaR) is said to have a heavier tail. The simulated results provided in Tables 2 and 3 show that the proposed model has higher values of the risk measures than the other competitive distributions.

Figure 4 displays the simulation results provided in Table 2; in addition, Figure 5 displays the simulation results provided in Table 3. 
Table 2: Simulation results for VaR abd TVaR of the NEx-APTW and other fitted distributions

\begin{tabular}{|c|c|c|c|c|}
\hline Distribution & Parameters & Level of significance & VaR & TVaR \\
\hline \multirow{8}{*}{ Weibull } & \multirow{8}{*}{$\begin{array}{l}\hat{\alpha}=0.5 \\
\hat{\gamma}=0.7\end{array}$} & 0.700 & 3.695 & 11.208 \\
\hline & & 0.750 & 4.674 & 12.617 \\
\hline & & 0.800 & 5.995 & 14.447 \\
\hline & & 0.850 & 7.885 & 16.970 \\
\hline & & 0.900 & 10.889 & 20.828 \\
\hline & & 0.950 & 16.885 & 28.199 \\
\hline & & 0.975 & 23.886 & 36.494 \\
\hline & & 0.999 & 67.955 & 85.868 \\
\hline \multirow{8}{*}{ EW } & \multirow{8}{*}{$\begin{array}{l}\hat{a}=0.5 \\
\hat{\alpha}=0.5 \\
\hat{\gamma}=0.7\end{array}$} & 0.700 & 1.414 & 3.076 \\
\hline & & 0.750 & 1.700 & 3.381 \\
\hline & & 0.800 & 2.060 & 3.758 \\
\hline & & 0.850 & 2.534 & 4.250 \\
\hline & & 0.900 & 3.217 & 4.949 \\
\hline & & 0.950 & 4.408 & 6.155 \\
\hline & & 0.975 & 5.614 & 7.369 \\
\hline & & 0.999 & 11.272 & 13.033 \\
\hline \multirow{8}{*}{ NEx-APTW } & \multirow{8}{*}{$\begin{array}{c}\hat{\alpha_{1}}=0.9 \\
\hat{\alpha}=0.5 \\
\hat{\gamma}=0.7 \\
\hat{\beta}=0.3\end{array}$} & 0.700 & 36.029 & 46.156 \\
\hline & & 0.750 & 37.733 & 48.016 \\
\hline & & 0.800 & 39.859 & 50.332 \\
\hline & & 0.850 & 42.665 & 53.380 \\
\hline & & 0.900 & 46.743 & 57.792 \\
\hline & & 0.950 & 54.038 & 65.646 \\
\hline & & 0.975 & 61.731 & 73.881 \\
\hline & & 0.999 & 102.339 & 116.836 \\
\hline
\end{tabular}

VaR = Value at Risk; TVaR = Tail VaR; EW = exponentiated Weibull; NEx-APTW = new extended alpha power transformed Weibull.
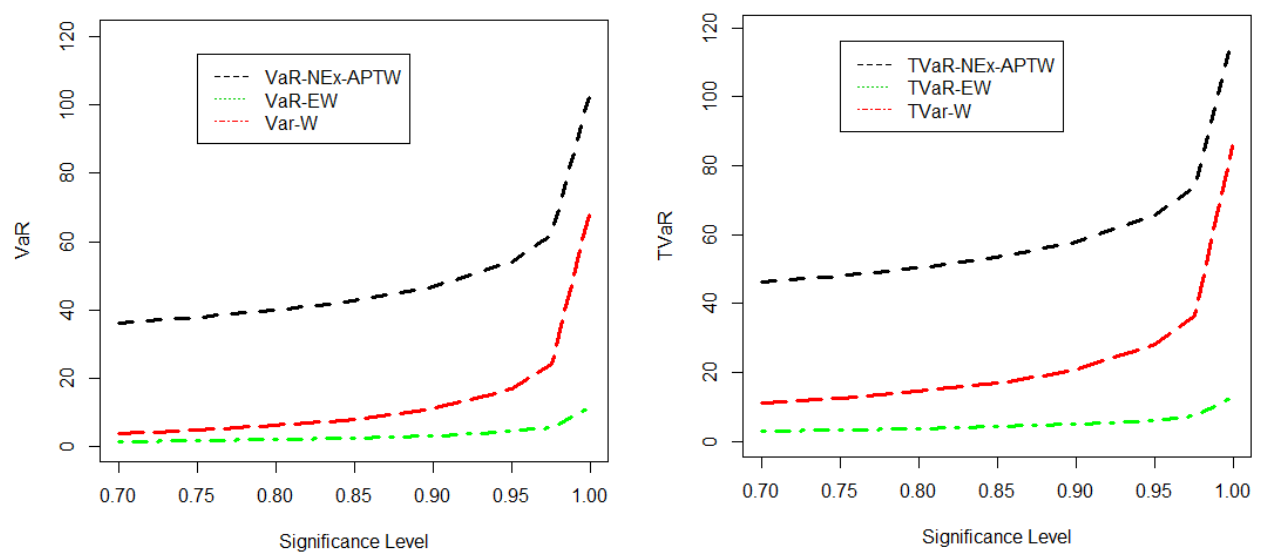

Figure 4: Graphical sketching of the results provided in Table 2. VaR = Value at Risk; TVaR = Tail VaR; $E W=$ exponentiated Weibull; NEX-APTW $=$ new extended alpha power transformed Weibull; $W=$ Weibull.

\section{An application to heavy tailed insurance data}

Actuaries are looking for new distributions to provide an adequate fit to heavy tailed data in actuarial, financial sciences and related areas. In this section, we analyze a real data set from insurance sciences to demonstrate the flexibility of the NEx-APTW distribution. We also calculate actual measures of the Weibull, EW, and NEx-APTW distributions using a real data set. 
Table 3: Simulation results for VaR abd TVaR of the NEx-APTW and other fitted distributions.

\begin{tabular}{|c|c|c|c|c|}
\hline Distribution & Parameters & Level of significance & VaR & TVaR \\
\hline \multirow{8}{*}{ Weibull } & \multirow{8}{*}{$\begin{array}{l}\hat{\alpha}=0.5 \\
\hat{\gamma}=0.7\end{array}$} & 0.700 & 3.601 & 15.695 \\
\hline & & 0.750 & 4.831 & 17.998 \\
\hline & & 0.800 & 6.593 & 21.084 \\
\hline & & 0.850 & 9.288 & 25.500 \\
\hline & & 0.900 & 13.905 & 32.569 \\
\hline & & 0.950 & 24.059 & 46.976 \\
\hline & & 0.975 & 37.119 & 64.354 \\
\hline & & 0.999 & 137.146 & 185.066 \\
\hline \multirow{8}{*}{ EW } & \multirow{8}{*}{$\begin{array}{l}\hat{a}=0.5 \\
\hat{\alpha}=0.5 \\
\hat{\gamma}=0.7\end{array}$} & 0.700 & 1.052 & 2.439 \\
\hline & & 0.750 & 1.286 & 2.694 \\
\hline & & 0.800 & 1.582 & 3.010 \\
\hline & & 0.850 & 1.976 & 3.424 \\
\hline & & 0.900 & 2.548 & 4.015 \\
\hline & & 0.950 & 3.554 & 5.039 \\
\hline & & 0.975 & 4.578 & 6.072 \\
\hline & & 0.999 & 9.398 & 10.902 \\
\hline \multirow{8}{*}{ NEx-APTW } & \multirow{8}{*}{$\begin{array}{c}\hat{\alpha_{1}}=0.9 \\
\hat{\alpha}=0.5 \\
\hat{\gamma}=0.7 \\
\hat{\beta}=0.3\end{array}$} & 0.700 & 56.131 & 78.680 \\
\hline & & 0.750 & 59.724 & 82.844 \\
\hline & & 0.800 & 64.265 & 88.080 \\
\hline & & 0.850 & 70.352 & 95.060 \\
\hline & & 0.900 & 79.371 & 105.338 \\
\hline & & 0.950 & 95.977 & 124.064 \\
\hline & & 0.975 & 114.069 & 144.267 \\
\hline & & 0.999 & 217.212 & 257.047 \\
\hline
\end{tabular}

VaR = Value at Risk; TVaR = Tail VaR; EW = exponentiated Weibull; NEx-APTW = new extended alpha power transformed Weibull.
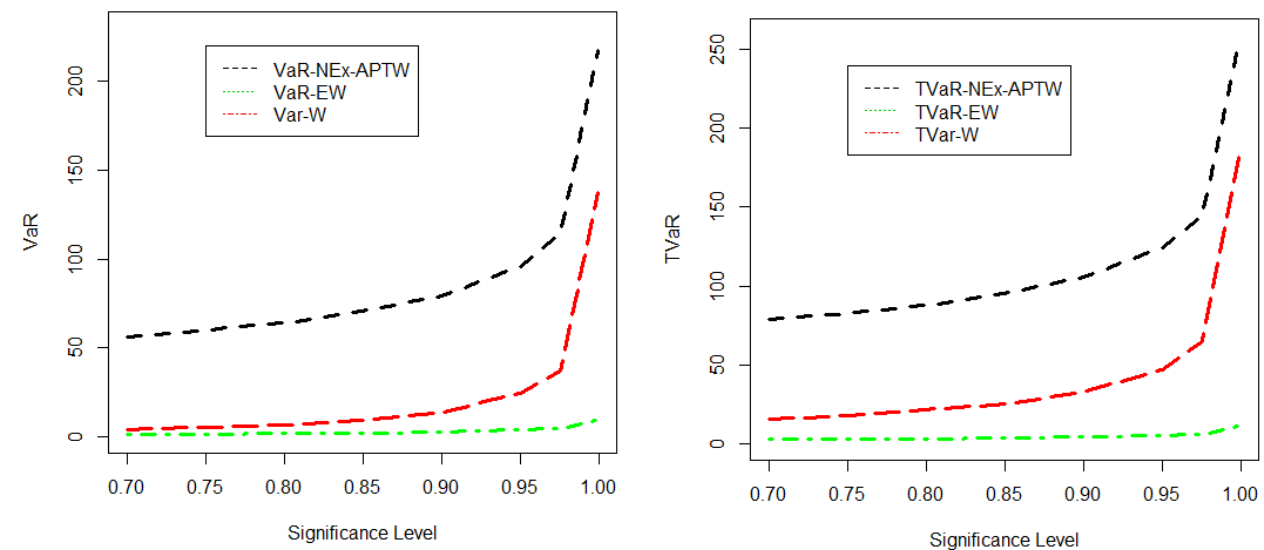

Figure 5: Graphical sketching of the results provided in Table 3. VaR = Value at Risk; TVaR = Tail VaR; $E W=$ exponentiated Weibull; NEx-APTW = new extended alpha power transformed Weibull.

\subsection{Hospital cost insurance data}

The data set representing hospital costs in the state of Wisconsin is published by the Office of the Health Care Information, Wisconsin's Department of Health and Human Resources. The data set is available at: https://www.dhs.wisconsin.gov/stats/index.htm. The comparison of the proposed method is made with the other ten (two, three and four parameters) well-known distributions. The cdf's of the 
competitive distributions are:

1. Weibull distribution

$$
G(x)=1-e^{-\gamma x^{\alpha}}, \quad x \geq 0, \alpha, \gamma>0 .
$$

2. Ex-APTW distribution

$$
G(x)=\frac{\alpha_{1}^{\left(1-e^{-\gamma x^{\alpha}}\right)}-e^{\left(1-e^{-\gamma x^{\alpha}}\right)}}{\alpha_{1}-e}, \quad x \geq 0, \alpha_{1}, \alpha, \gamma>0, \alpha_{1} \neq e .
$$

3. Lomax distribution

$$
G(x)=1-(1+\gamma x)^{-\alpha}, \quad x \geq 0, \alpha, \gamma>0 .
$$

4. Burr-XII (B-XII) distribution

$$
G(x)=1-\left(1+x^{\gamma}\right)^{-\alpha}, \quad x \geq 0, \alpha, \gamma>0 .
$$

5. Pareto distribution

$$
G(x)=1-\left(\frac{\gamma}{x}\right)^{\alpha}, \quad x \geq 0, \alpha, \gamma>0
$$

6. The alpha power transformed Weibull (APTW) distribution

$$
G(x)=\frac{\alpha_{1}^{\left(1-e^{-\gamma x^{\alpha}}\right)}-1}{\alpha_{1}-1}, \quad x \geq 0, \alpha_{1}, \alpha, \gamma>0, \alpha_{1} \neq 1 .
$$

7. The Marshall-Olkin (MOW) distribution

$$
G(x)=\frac{\left(1-e^{-\gamma x^{\alpha}}\right)}{\sigma+(1-\sigma)\left(1-e^{-\gamma x^{\alpha}}\right)}, \quad x \geq 0, \alpha, \gamma, \sigma>0 .
$$

8. EW distribution

$$
G(x)=\left(1-e^{-\gamma x^{\alpha}}\right)^{a}, \quad x \geq 0, \alpha, \gamma, a>0 .
$$

9. Kumaraswamy Weibull $(\mathrm{Ku}-\mathrm{W})$ distribution

$$
G(x)=1-\left\{1-\left(1-e^{-\gamma x^{\alpha}}\right)^{a}\right\}^{b}, \quad x \geq 0, \alpha, \gamma, a, b>0 .
$$

10. The beta Weibull (BW) distribution

$$
G(x)=I_{\left(1-e^{-\gamma x^{\alpha}}\right)}(a, b), \quad x \geq 0, \alpha, \gamma, a, b>0 .
$$


Table 4: Estimated values with standard error (in parenthesis) of the proposed and other competitive models for the hospital cost insurance data

\begin{tabular}{|c|c|c|c|c|c|c|c|}
\hline Distribution & $\overline{\overline{\alpha_{1}}}$ & $\overline{\hat{\alpha}}$ & $\overline{\hat{\gamma}}$ & $\overline{\bar{\sigma}}$ & $\overline{\hat{\beta}}$ & $\overline{\bar{a}}$ & $\bar{b}$ \\
\hline NEx-APTW & $3.419(2.751)$ & $0.696(0.028)$ & $1.469(0.231)$ & & $0.521(1.613)$ & & \\
\hline Ex-APTW & $3.893(2.751)$ & $0.865(0.183)$ & $1.665(0.069)$ & & & & \\
\hline Weibull & & $0.674(0.022)$ & $1.918(0.084)$ & & & & \\
\hline Lomax & & $1.569(0.207)$ & $0.365(0.074)$ & & & & \\
\hline B-XII & & $2.885(0.129)$ & $0.813(0.026)$ & & & & \\
\hline Pareto & & $2.970(0.420)$ & $1.909(2.895)$ & & & & \\
\hline APTW & $0.185(2.910)$ & $1.229(0.403)$ & $0.762(0.945)$ & & & & \\
\hline MOW & & $0.808(0.955)$ & $1.186(0.863)$ & $0.387(0.145)$ & & & \\
\hline EW & & $1.944(0.459)$ & $2.564(0.261)$ & & & $0.487(0.063)$ & \\
\hline $\mathrm{Ku}-\mathrm{W}$ & & $0.847(0.805)$ & $1.086(1.290)$ & & & $2.567(2.906)$ & $4.583(1.095)$ \\
\hline BW & & $0.441(0.089)$ & $0.828(0.928)$ & & & $2.097(0.761)$ & $3.804(1.092)$ \\
\hline
\end{tabular}

NEx-APTW = new Ex-APTW; Ex-APTW = extended APTW; B-XII = Burr-XII; APTW = alpha power transformed Weibull; MOW = Marshall-Olkin; EW = exponentiated Weibull; Ku-W = Kumaraswamy Weibull; BW = beta Weibull.

To determine the goodness-of-fit among the applied distributions, we consider certain goodnessof-fit measures such as Cramer-Von-Messes (CM) test statistic, Anderson Darling (AD) test statistic and Kolmogorov-Simonrove (KS) test statistic with corresponding $p$-value. The measure are given by:

- The AD test statistic

$$
\mathrm{AD}=-n-\frac{1}{n} \sum_{i=1}^{n}(2 i-1)\left[\log G\left(x_{i}\right)+\log \left\{1-G\left(x_{n-i+1}\right)\right\}\right],
$$

where $n=$ the sample size, $x_{i}=$ the $i^{\text {th }}$ sample, calculated when the data is sorted in ascending order.

- The CM test statistic

$$
\mathrm{CM}=\frac{1}{12 n}+\sum_{i=1}^{n}\left[\frac{2 i-1}{2 n}-G\left(x_{i}\right)\right]^{2} .
$$

- The KS test statistic is given by

$$
\mathrm{KS}=\sup _{x}\left[G_{n}(x)-G(x)\right]
$$

where $G_{n}(x)$ is the empirical cdf and $\sup _{x}$ is the supremum of the set of distances.

A distribution with lower values of these analytical measures is considered as a good candidate model among the applied distributions for the underlying data sets. Based on the considered measures, the NEx-APTW distribution has the lowest values among all fitted models for the hospital cost insurance data. Table 4 reports parameter values with standard errors in parenthesis. In support of the numerical measures provided in Table 5, the empirical cdf and sf of the NEx-APTW are plotted in Figure 6. From Figure 6, we can see that the proposed model fit empirical cdf and sf very closely. In addition, the PP plot of the NEx-APTW distribution for the respective data set is plotted in Figure 7 and shows that the proposed provide best fit to the considered data. The box plot of the data set is also sketched in Figure 7 showing that the hospital cost insurance data is skewed to the right. 
Table 5: Goodness-of-fit measures of the proposed and other competitive models for the hospital cost insurance data

\begin{tabular}{ccccc}
\hline \hline Distribution & CM & AD & KS & $p$-value \\
\hline NEx-APTW & 0.093 & 0.890 & 0.031 & 0.695 \\
Ex-APTW & 0.109 & 0.932 & 0.034 & 0.585 \\
Weibull & 0.112 & 0.936 & 0.035 & 0.035 \\
Lomax & 0.464 & 2.623 & 0.078 & 0.002 \\
B-XII & 0.250 & 1.426 & 0.045 & 0.229 \\
Pareto & 0.560 & 1.708 & 0.058 & 0.247 \\
APTW & 0.152 & 0.957 & 0.038 & 0.414 \\
MOW & 0.155 & 0.961 & 0.037 & 0.447 \\
EW & 0.168 & 0.997 & 0.039 & 0.150 \\
Ku-W & 0.159 & 0.944 & 0.197 & 0.396 \\
BW & 1.948 & 1.912 & 0.206 \\
\hline \hline
\end{tabular}

CM = Cramer-Von-Messes; AD = Anderson Darling; KS = Kolmogorov-Simonrove; NEx-APTW = new Ex-APTW; ExAPTW = extended APTW; B-XII = Burr-XII; APTW = alpha power transformed Weibull; MOW = Marshall-Olkin; EW = exponentiated Weibull; Ku-W = Kumaraswamy Weibull; $\mathrm{BW}=$ beta Weibull.
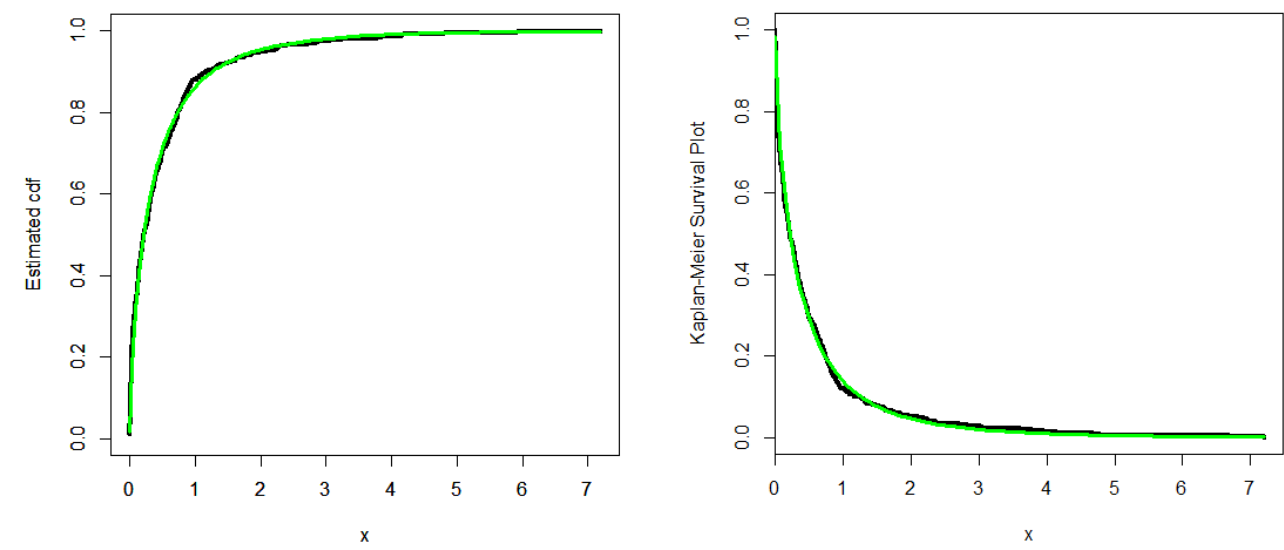

Figure 6: Estimated cdf and Kaplan-Meier survival plots of the new extended alpha power transformed Weibull distribution for the hospital cost insurance data.

\subsection{Computation of actuarial measures using real data set}

In this sub-section, we compute VaR and TVaR measures of Weibull, EW and the NEx-APTW distributions using estimated parameters values analyzed in Subsection 7.1. Table 6 reports the numerical results. A model with higher values of the risk measures possesses the heavier tails. The numerical results for the actuarial measures of the proposed and the other distributions show that the proposed distribution has a heavier tail than Weibull and EW distributions. In addition, it can be used as a good candidate model for modeling heavy tailed insurance data sets.

\section{Concluding remarks}

In this article, a new family of distributions called a new extended alpha power transformed family has been proposed. The proposed method examines a four-parameter special model of a new extended alpha power transformed Weibull distribution. Actuarial measures of the proposed model are also calculated and a simulation study is conducted to show the usefulness of the proposed method in 

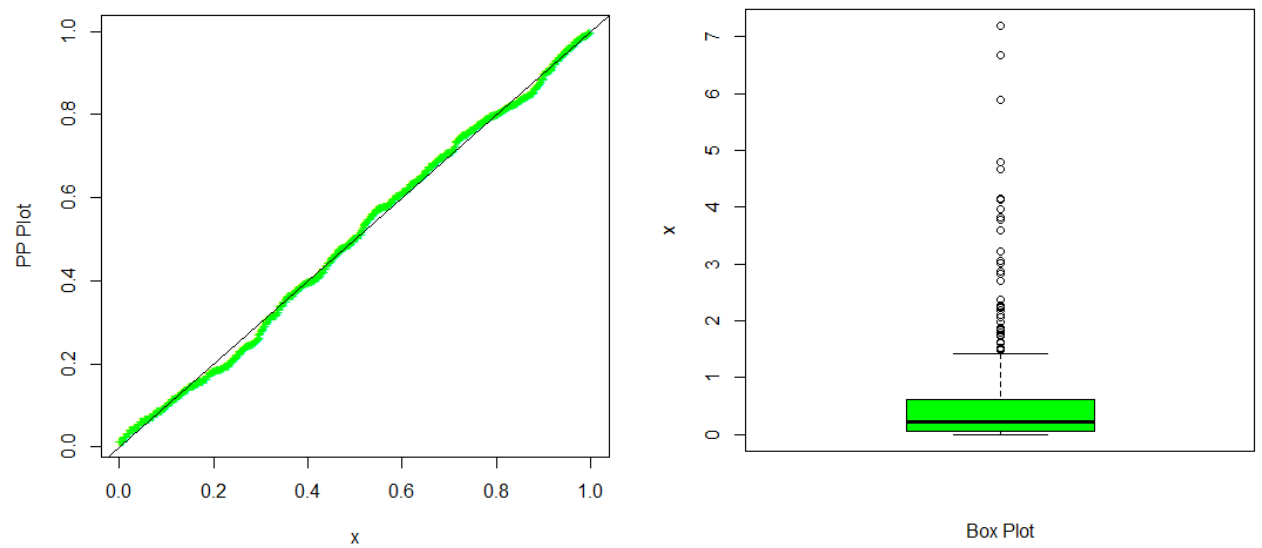

Figure 7: PP plot of the new extended alpha power transformed Weibull distribution and box plot for the hospital cost insurance data.

Table 6: Results for the actuarial measures using health care insurance data

\begin{tabular}{ccccc}
\hline \hline Distribution & Parameters & Level of significance & VaR & TVaR \\
\hline \multirow{5}{*}{ Weibull } & & 0.700 & 0.710 & 0.914 \\
& & 0.750 & 1.047 & 1.275 \\
& $\hat{\alpha}=0.674$ & 0.800 & 1.323 & 1.709 \\
& $\hat{\gamma}=1.918$ & 0.850 & 1.793 & 1.907 \\
& & 0.900 & 2.134 & 2.086 \\
& & 0.950 & 2.600 & 2.395 \\
& & 0.975 & 2.906 & 2.706 \\
EW & 0.999 & 3.145 & 2.875 \\
\hline & & 0.700 & 0.023 & 0.021 \\
& $\hat{a}=0.487$ & 0.750 & 0.108 & 0.067 \\
& $\hat{\alpha}=1.944$ & 0.800 & 0.290 & 0.153 \\
& $\hat{\gamma}=2.564$ & 0.850 & 0.787 & 0.490 \\
& & 0.900 & 0.973 & 0.630 \\
& & 0.950 & 1.122 & 0.959 \\
& & 0.975 & 1.645 & 1.141 \\
& & 0.999 & 1.760 & 1.397 \\
\hline \multirow{5}{*}{ NEx-APTW } & 0.700 & 0.925 & 1.563 \\
& $\hat{\alpha}=3.419$ & 0.750 & 1.754 & 2.901 \\
& $\hat{\alpha}=0.696$ & 0.800 & 1.895 & 3.396 \\
& $\hat{\gamma}=1.469$ & 0.850 & 2.365 & 5.303 \\
& $\hat{\beta}=0.521$ & 0.900 & 3.933 & 5.925 \\
& & 0.950 & 4.884 & 6.964 \\
& & 0.975 & 5.510 & 7.313 \\
& & 0.999 & 6.339 & 7.890 \\
\hline
\end{tabular}

VaR = Value at Risk; TVaR = Tail VaR; EW = exponentiated Weibull; NEx-APTW = new extended alpha power transformed Weibull.

actuarial sciences. A practical application to the heavy tailed insurance data is analyzed and the comparison of the proposed model with the other nine well-known competitors are presented. Actuarial measures based on a real data set is also calculated which shows that the proposed model may be a good candidate model to analyze actuarial data sets. We hope that the proposed method will attract a wider applications in actuarial sciences and related fields. 


\section{Data availability statement}

This work is a methodological development and has been applied on secondary data related to the hospital cost insurance data if required, data can be provided to interested readers.

\section{Acknowledgments}

The authors are grateful to the Editor-in-Chief, the Associate Editor and anonymous referees for their valuable comments and suggestions that helped improve the manuscript. The first two authors also acknowledge the support of Yazd University, Iran.

\section{Dedication}

This article is drafted from the Ph.D. work of the first author who would like to dedicate this article to the memory of his late parents.

\section{References}

Adcock C, Eling M, and Loperfido N (2015). Skewed distributions in finance and actuarial science: a review, The European Journal of Finance, 21, 1253-1281.

Ahmad Z, Hamedani GG, and Butt NS (2019a). Recent developments in distribution theory: a brief survey and some new generalized classes of distributions, Pakistan Journal of Statistics and Operation Research, 15, 87-110.

Ahmad Z, Ilyas M, and Hamedani GG (2019b). The extended alpha power transformed family of distributions: properties and applications, Journal of Data Science, 17, 726-741.

Artzner P (1999). Application of coherent risk measures to capital requirements in insurance, North American Actuarial Journal, 3, 11-25.

Bagnato L and Punzo A (2013). Finite mixtures of unimodal beta and gamma densities and the $k$ bumps algorithm, Computational Statistics, 28, 1571-1597.

Bakar SA, Hamzah NA, Maghsoudi M, and Nadarajah S (2015). Modeling loss data using composite models, Insurance: Mathematics and Economics, 61, 146-154.

Bernardi M, Maruotti A, and Petrella L (2012). Skew mixture models for loss distributions: a Bayesian approach, Insurance: Mathematics and Economics, 51, 617-623.

Bhati D and Ravi S (2018). On generalized log-Moyal distribution: A new heavy tailed size distribution, Insurance: Mathematics and Economics, 79, 247-259.

Cooray K and Ananda MM (2005). Modeling actuarial data with a composite lognormal-Pareto model, Scandinavian Actuarial Journal, 2005, 321-334.

Eling M (2012). Fitting insurance claims to skewed distributions: Are the skew-normal and skewstudent good models?, Insurance: Mathematics and Economics, 51, 239-248.

García VJ, Gómez-Déniz E, and Vázquez-Polo FJ (2014). On modelling insurance data by using a generalized lognormal distribution, Revista de Métodos Cuantitativos para la Economía y la Empresa, 18, 146-162.

Glänzel W (1987). A characterization theorem based on truncated moments and its application to some distribution families. In Mathematical Statistics and Probability Theory (pp. 75-84), Springer, Dordrecht.

Glänzel W (1990). Some consequences of a characterization theorem based on truncated moments, Statistics, 21, 613-618.

Ibragimov R and Prokhorov A (2017). Heavy tails and copulas: topics in dependence modelling in 
economics and finance.

Kazemi R and Noorizadeh M (2015). A comparison between skew-logistic and skew-normal distributions, MATEMATIKA: Malaysian Journal of Industrial and Applied Mathematics, 31, 15-24.

Klugman SA, Panjer HH, and Willmot GE (2012). Loss Models: From Data to Decisions (4th ed), John Wiley and Sons, Hoboken, NJ.

Landsman Z, Makov U, and Shushi T (2016). Tail conditional moments for elliptical and log-elliptical distributions, Insurance: Mathematics and Economics, 71, 179-188.

Lane MN (2000). Pricing risk transfer transactions 1, ASTIN Bulletin: The Journal of the IAA, 30, 259-293.

Mahdavi A and Kundu D (2017). A new method for generating distributions with an application to exponential distribution, Communications in Statistics-Theory and Methods, 46, 6543-6557.

Punzo A, Bagnato L, and Maruotti A (2018). Compound unimodal distributions for insurance losses, Insurance: Mathematics and Economics, 81, 95-107.

Reynkens T, Verbelen R, Beirlant J, and Antonio K (2017). Modelling censored losses using splicing: A global fit strategy with mixed Erlang and extreme value distributions, Insurance: Mathematics and Economics, 77, 65-77.

Received September 19, 2019; Revised December 15, 2019; Accepted January 10, 2020 
